\title{
Entropy Production in a Non-Isolated Thermodynamic System Taking into Account Regular Factors of Nonrandom Nature
}

\author{
A. Yu. Khlestkov, Yu. A. Khlestkov, N. Yu. Lukashina, M. Yu. Lukashin, P. Yu. Lukashin \\ National Research Nuclear University MEPhI, Moscow, Russia \\ Email: khlestkov@yandex.ru
}

How to cite this paper: Khlestkov, A.Y., Khlestkov, Y.A., Lukashina, N.Y., Lukashin, M.Y. and Lukashin, P.Y. (2020) Entropy Production in a Non-Isolated Thermodynamic System Taking into Account Regular Factors of Nonrandom Nature. Journal of Modern Physics, 11, 343-354. https://doi.org/10.4236/jmp.2020.113021

Received: January 23, 2020

Accepted: February 28, 2020

Published: March 2, 2020

Copyright ( 2020 by author(s) and Scientific Research Publishing Inc. This work is licensed under the Creative Commons Attribution International License (CC BY 4.0).

http://creativecommons.org/licenses/by/4.0/ (c) (i) Open Access

\begin{abstract}
The work illustrates the impossibility of decreasing entropy in a strictly random thermodynamic process in a non-isolated system using the example of heating a planet by solar radiation flux without and taking into account its rotation around its own axis. That is, the second law of thermodynamics formulated for isolated systems continues to govern such systems. We have shown that in order to achieve a stationary state at lower values of temperature and entropy far from thermodynamic equilibrium at a maximum of temperature and entropy, it is necessary to have regular factors of nonrandom nature, one of which in this example is the rotation of the planet around its own axis. This means that the reason for the appearance of ordered structured objects in non-isolated thermodynamic systems is not the random process itself, but the action of dynamic control mechanisms, such as periodic external influences, nonlinear elements with positive feedback, catalysts for chemical reactions, etc. We present the plots with dependences of temperature and entropy versus time in non-isolated systems with purely random processes and in the presence of a control factor of non-random nature-rotation.
\end{abstract}

\section{Keywords}

Random Process, Non-Isolated Systems, Entropy, Ordered Structures, Regular Factors of Non-Random Nature

\section{Introduction}

When studying the problem of origin of ordered structures: elementary particles, nuclei of atoms, crystals, biomolecules, cells, ..., basically one model is consi- 
dered: their occurrence in a random process [1] [2] [3]. With this "self-organization" the disorder should decrease. This means that entropy as a measure of disorder in a thermodynamic process should decrease. But the second law of thermodynamics prohibits a decrease in entropy in random processes in isolated systems. Therefore, our certain hopes are associated with the openness of real systems allowing the presence of ingoing and outgoing flows of heat (closed systems), heat and particles (open systems) that do not fall under action of the second law. It is believed that there it is possible, in principle, to reduce entropy due to its "removal" by the outgoing flows, and, therefore, the creation of conditions for the appearance of ordered structures in a random process.

We consider the well-known "entropy pump" model which describes the process of decreasing entropy in the Earth's ecosphere. In this model, a change in the entropy $\mathrm{S}$ with a speed $\mathrm{d} S / \mathrm{d} t$ occurs in a non-isolated system in the course of a stationary process, when the ingoing ordered low-entropy heat flow $P_{s}$ at solar temperature $T_{s}$ is compared in equilibrium with the outgoing flow $P=P_{s}$. The latter is already more randomized (highly entropic) and having a lower temperature $T<T_{s}$. In this case, the rate of change in the entropy of the system becomes negative [4]:

$$
\frac{\mathrm{d} S}{\mathrm{~d} t}=P_{s}\left(\frac{1}{T_{s}}-\frac{1}{T}\right)<0
$$

There are two comments regarding formula (1). Firstly, concerning a stationary process in a homogeneous system, which in this example is a planet (neglecting the atmosphere) heated by the solar radiation flux $P_{s}$, when two temperatures arise, $T_{s}$ and $T<T_{s}$. Since temperature is a function of the state, then in an equilibrium thermodynamic state the system can have only one temperature equal to the temperature of the ingoing heat flow $T_{s}$.

Secondly, the entropy $S$ itself is also a function of the state, therefore, in equilibrium state it must also be constant, that is, its rate of change $\mathrm{d} S / \mathrm{d} t$ must be zero.

Let's see how we can obtain formula (1), and what it means in the classical thermodynamics of quasistatic systems, i.e. those in which a local thermodynamic equilibrium can be established in a non-equilibrium process in a small neighborhood of each point.

The simplest model was used (Figure 1), which we will describe in detail below. As a result of solving her system of differential equations, the following results were obtained:

- Taken with the opposite sign, formula (1) describes in fact the usually neglected production of entropy at the input to the system at the boundary of a body and the environment during thermalization of the ingoing heat flow $P_{s}$ and its randomization during interaction between the photons belonging to the ingoing solar radiation and atoms of the crystalline lattice at the surface of the system (the planet's soil, in this example); 


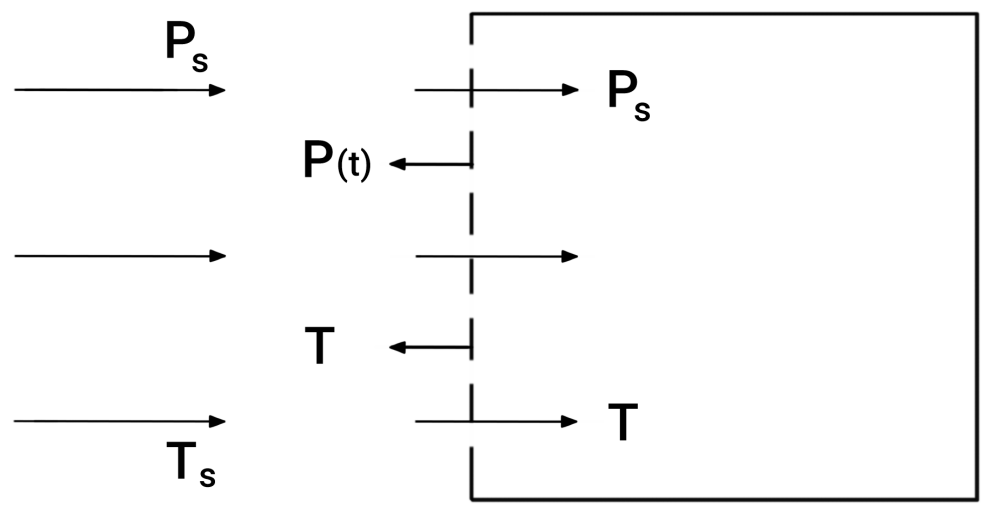

Figure 1. A non-isolated thermodynamic system with an input point outside the body. Thermalization of the ingoing heat flow at the system boundary.

- Taking into account this production of entropy, despite its removal by the outgoing heat flow during the heating of the planet, the entropy of this non-isolated system does not decrease. That is, the second law of thermodynamics remains valid for non-isolated homogeneous systems, if all the processes occurring in it are random;

- At $t \rightarrow \infty, T \rightarrow T_{s}, \frac{\mathrm{d} S}{\mathrm{~d} t} \rightarrow 0, S(t) \rightarrow S_{\infty \text { max }}$;

that is, a new asymptotic equilibrium state of the system is achieved at the maximum temperature $T=T_{s}$ and the entropy $S=S_{\infty \max }$. There is no entropy pump here.

But a paradox arises: in fact, however, real planets without a significant atmosphere (such as Mercury, Mars, and Pluto) really have two temperatures: $T_{s}$ is the total radiation temperature of ingoing solar radiation and $T_{\infty}<T_{s}$ is the stationary temperature of the planet surface layer heated by solar heat. Therefore, they are not described by this thermodynamic model.

It is clear, what is the reason for this difference. In the considered illustrative example, the influence of the planet's rotation around its axis on the temperature and entropy balance is not taken into account. Therefore, we have a periodic change of day and night, at which heating, simultaneous emission of heat by the body and an increase in entropy occur on the day side of the planet, while on its night side the planet only radiates heat without generating entropy.

This leads to the fact that the stationary state is achieved by a non-isolated system at lower values of temperature and entropy, far from the thermodynamic maximum.

We took into account this rotation (in the general case, a periodic pulsed heat flow from the heater (Figure 2)) and we have obtained the expected result: rotation of the planet around its axis decreases the stationary temperature of its surface and entropy.

The calculation of the day and night asymptotic temperature values $T_{d \infty}$ and $T_{n \infty}$ for the planets mentioned above showed good agreement with the experimental data (see Table 1). 
Table 1. Calculation of the Day and Night temperatures for the three planets.

\begin{tabular}{|c|c|c|c|}
\hline Planet & Mercury & Mars & Pluto \\
\hline Distance from the Sun, million $\mathrm{km}$ (a.u.) & $57(0.39)$ & $230(1.5)$ & $6000(40)$ \\
\hline Solar constant, $\mathrm{W} / \mathrm{m}^{2}$ & 4200 & 590 & 1 \\
\hline Rotation period, Earth days & 58.6 & 1.03 & 6.39 \\
\hline Total radiation temperature due to solar radiation, $\mathrm{K}$ & 630 & 320 & 63 \\
\hline Daily experimental temperature $T_{d \infty}, \mathrm{K}$ & $500-700$ & $200-300$ & 40 \\
\hline Daily calculated temperature $T_{d \infty}, \mathrm{K}$ & 630 & 270 & 50 \\
\hline Night experimental temperature $T_{n \infty}, \mathrm{K}$ & 100 & $150-250$ & - \\
\hline Night calculated temperature $T_{n \infty}, \mathrm{K}$ & 150 & 260 & 50 \\
\hline
\end{tabular}

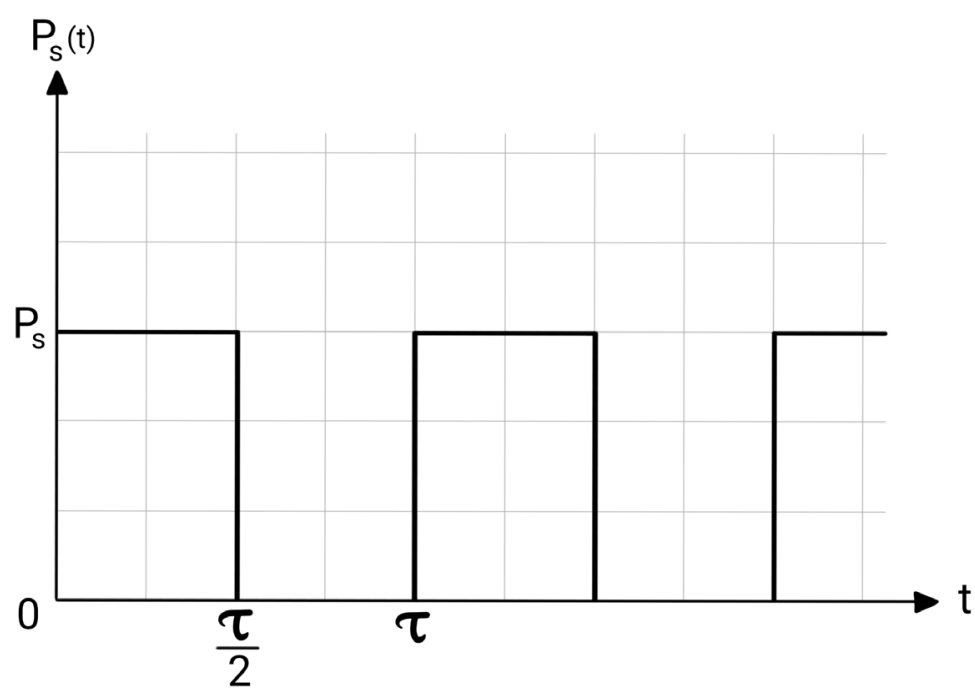

Figure 2. The periodic heat flow $P_{s}(t)$ as the regular control factor.

Thus, in addition to the conclusion that entropy still does not decrease in a non-isolated system with purely random processes, which means that no self-organization phenomena are possible in purely random processes, one more result is obtained: the entropy of a stationary non-isolated system can become less than a thermodynamic maximum only under the influence of regular factors of a non-random dynamic nature (in this example, pulsed semi-periodic heat transfer, starting from $t=0$ ).

Hence the following conclusion is possible: the reason for the decrease in disorder and the appearance of structured objects is not the random process itself, but the action of not random ordering mechanisms, moreover of external origin: periodic external influences, such as nuclear or chemical reactions, nonlinear dynamic mechanisms with positive feedback, etc.

This result is consistent with the Prigogine hypothesis about the existence of control parameters [1] [3]. We only note according to the result, that these parameters can be of a regular nature, and their nature needs to be investigated.

For example, the considered mechanism of periodic interruption of the in- 
going heat flow is provided by the rotation of the planets during the gravitational interaction of bodies in the universe.

\section{Thermodynamic Model without Rotation}

In Figure 1, a low-entropic heat flow $P_{s}$ with a total radiation temperature $T_{s}$ falls on the boundary of a non-isolated system and becomes randomized when interacting with the surface of a body (planet). Other dissipative processes (thermal conductivity, diffusion, viscosity ...) are neglected.

This heat flow begins to heat the body from the initial temperature $T_{0}$ to $T(t)$. When heated, the body begins to radiate a return heat flow into an external environment with increasing power $P(t)$ until a new equilibrium state with the entropy $S(T)$ is reached, which, like the body temperature $T(t)$, will be calculated from the following balance of entropy [5] [6]:

$$
\begin{aligned}
\frac{\mathrm{d} S}{\mathrm{~d} t} & =\frac{\mathrm{d} S_{\text {ext }}}{\mathrm{d} t}+\frac{\mathrm{d} S_{\text {int }}}{\mathrm{d} t}=\left(\frac{\mathrm{d} S_{\text {inp }}}{\mathrm{d} t}-\frac{\mathrm{d} S_{\text {out }}}{\mathrm{d} t}\right)+\frac{\mathrm{d} S_{\text {int }}}{\mathrm{d} t} \\
& =\left(\frac{P_{s}}{T_{s}}-\frac{P(t)}{T}\right)+\left(\frac{P_{s}}{T}-\frac{P_{s}}{T_{s}}\right)=\frac{1}{T} \frac{\mathrm{d} U+\delta A}{\mathrm{~d} t} \\
& =\frac{1}{T} C_{v} M \frac{\mathrm{d} T}{\mathrm{~d} t}+\frac{1}{T} \frac{\delta A}{\mathrm{~d} t}
\end{aligned}
$$

Here $S_{\text {ext }}$ is the entropy transferred by heat flows: ingoing, $S_{\text {inp }}$, with speed $\frac{P_{s}}{T_{s}}$, and outgoing, $S_{\text {out }}$ with speed $\frac{P(T)}{T} ; S_{\text {int }}$ is the entropy produced inside the system during thermalization of the ingoing heat flow at a speed equal to the increment $\left(\frac{P_{s}}{T}-\frac{P_{s}}{T_{s}}\right) ; \mathrm{d} U$ is the differential of internal energy, $c_{V}$ is the heat capacity of a body with a heated mass $M$; $\delta A$ is a work performed by the body in the process of thermalization of the ingoing heat flow.

For simplicity, we assume that all heat flows are radiated by a completely black body:

$$
\begin{aligned}
P_{s} & =\Pi \sigma T_{s}^{4}, \\
P & =\Pi \sigma T^{4},
\end{aligned}
$$

where $\Pi$-surface area of the heated body, $\sigma$-Stefan-Boltzmann constant.

From relations (2)-(4), it follows:

$$
\begin{aligned}
\frac{\mathrm{d} S_{\text {ext }}}{\mathrm{d} t} & =\Pi \sigma\left(T_{s}^{3}-T^{3}\right)=c_{v} M \frac{1}{T} \frac{\mathrm{d} T}{\mathrm{~d} t}, \\
\frac{\mathrm{d} S_{\text {int }}}{\mathrm{d} t} & =P_{s}\left(\frac{1}{T}-\frac{1}{T_{s}}\right)=\frac{1}{T} \frac{\delta A}{\mathrm{~d} t}, \\
\frac{\mathrm{d} S}{\mathrm{~d} t} & =\frac{\Pi \sigma}{T}\left(T_{s}^{4}-T^{4}\right) .
\end{aligned}
$$

From (5) we obtain the equation for $T(t)$ : 


$$
\frac{\Pi \sigma}{C_{v} M} \mathrm{~d} t=\frac{1}{T} \frac{\mathrm{d} T}{T_{s}^{3}-T^{3}}
$$

and from (7), substituting into it the time differential $\mathrm{d} t$ from (8), we obtain the equation for $S(T)$ :

$$
\mathrm{d} S=c_{v} M \frac{T_{s}^{4}-T^{4}}{T^{2}\left(T_{s}^{3}-T^{3}\right)} \mathrm{d} T .
$$

For convenience, we introduce dimensionless quantities (with a tilde)

$$
\begin{array}{r}
\mathrm{d} t=\frac{\tau}{2} \mathrm{~d} \tilde{t}, \\
\mathrm{~d} T=T_{\mathrm{s}} \mathrm{d} \tilde{T}, \\
\mathrm{~d} S=c_{v} M \mathrm{~d} \tilde{S} .
\end{array}
$$

Substituting (10) into (8) and (9), we finally obtain the differential equations for $\tilde{T}(\tilde{t})$ and $\tilde{S}(\tilde{T})$ in the dimensionless form:

$$
\begin{gathered}
\alpha \mathrm{d} \tilde{t}=\frac{1}{\tilde{T}} \frac{\mathrm{d} \tilde{T}}{1-\tilde{T}^{3}}, \\
\mathrm{~d} \tilde{S}=\frac{1}{\tilde{T}^{2}} \frac{1-\tilde{T}^{4}}{1-\tilde{T}^{3}} \mathrm{~d} \tilde{T},
\end{gathered}
$$

where the dimensionless constant is

$$
\alpha=\frac{\Pi \sigma \tau}{2 c_{v} M} T_{s}^{3} .
$$

\section{Non-Isolated System without Rotation}

Integrating Equation (11), Equation (12) with the initial conditions:

$$
\tilde{t}=\tilde{t}_{0}, \tilde{T}=\tilde{T}_{0}, \tilde{S}=\tilde{S}_{0}\left(t_{0}\right)
$$

and omitting hereinafter all tildes for dimensionless temperatures $T(t)$ and entropy increment:

$$
\Delta S(t)=S(t)-S_{0}\left(t_{0}\right)
$$

we get the following solutions:

$$
\begin{gathered}
T(t)=\left(1-\left(T_{0}^{-3}-1\right) \mathrm{e}^{-3 \alpha\left(t-t_{0}\right)}\right)^{-\frac{1}{3}} \\
\Delta S(T)=\frac{1}{2}\left(\ln \frac{1+T+T^{2}}{1+T_{0}+T_{0}^{2}}+2\left(\frac{1}{T_{0}}-\frac{1}{T}\right)-\frac{2}{\sqrt{3}}\left(\operatorname{arctg} \frac{2 T+1}{\sqrt{3}}-\operatorname{arctg} \frac{2 T_{0}+1}{\sqrt{3}}\right)\right) .
\end{gathered}
$$

Equation (11), Equation (13) and their solutions (16), (17) are valid within the framework of the classical nonrelativistic thermodynamics for any non-isolated homogeneous systems in which thermodynamic random processes occur. But in the capacity of an illustrative example explaining the meaning of the quantities introduced in the model, we consider heating a planet of mass $M$ with specific heat capacity of the heated layer $c_{V}$ with surface area $\Pi$ by solar radiation with a heat flow $P_{s}$ and with a total radiation temperature at the input to the system $T_{s}$, which is then reradiated by the planet into the surrounding vacuum by the heat 
flow $P(T)$ at a changing temperature $T(t)$ until a new equilibrium state is reached at $t \rightarrow \infty$.

From the solutions (16), (17) it directly follows that both the temperature $T(t)$ and the entropy $S(T(t))$ are monotonically increasing functions which depend on time $t$, reaching their equilibrium values $T_{\infty}$ and $S_{\infty}$ in the asymptotics $t \rightarrow \infty$ corresponding to the thermodynamic maximum (Figure 3, Figure 4, curves at $\omega=0$ ).

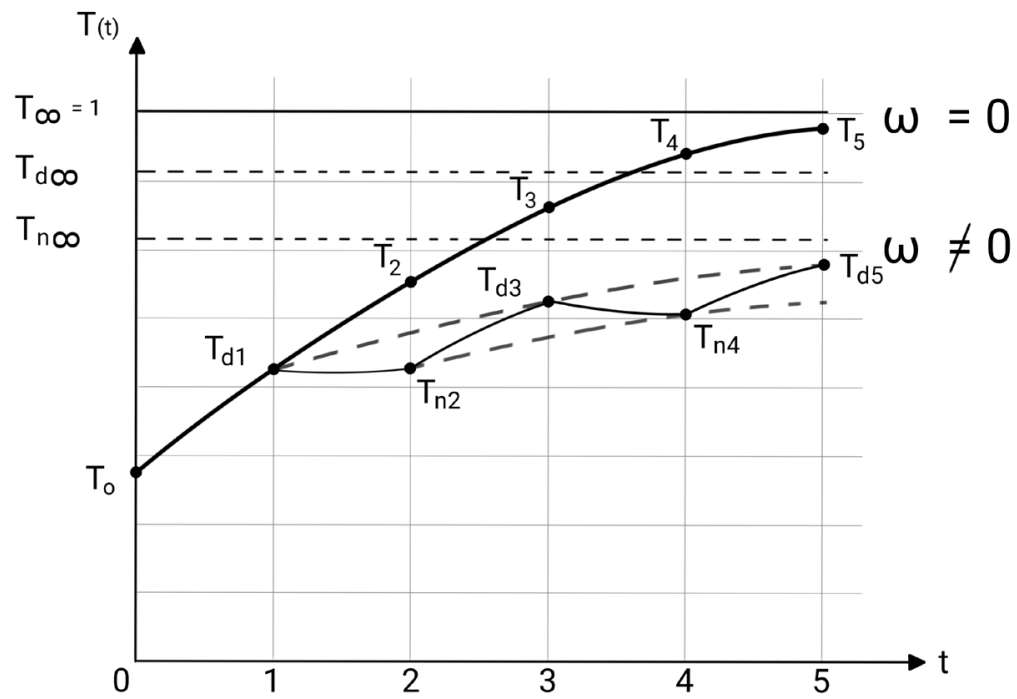

Figure 3. The dependence of the dimensionless body (planet) temperature $T\left(t, T_{0}\right)$ of a non-isolated thermodynamic system in the absence of rotation $(\omega=0)$ and in the presence of rotation $(\omega \neq 0)$ (periodic heat flow at the input) on the day and night sides.

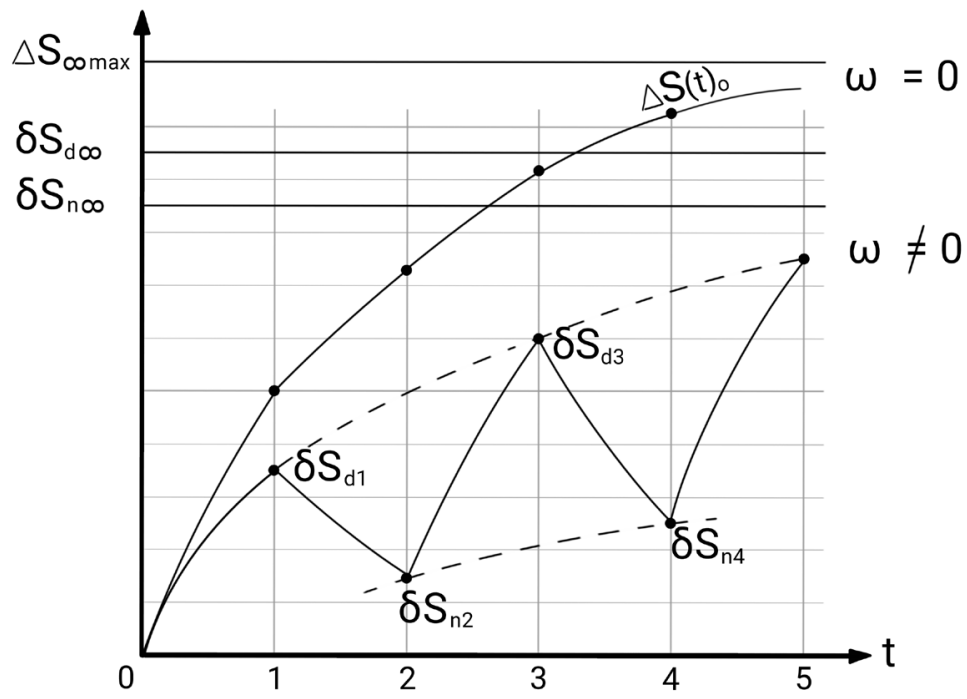

Figure 4. The entropy increment $\Delta S$ of a non-isolated system in a random process $(u=0$, there is no rotation as a control factor) and increments of entropy on the day and night sides of the planet relative to its initial value $S_{0}\left(T_{0}\right)$ in the presence of a control factor of non-random nature (planet rotation around its axis, $\omega \neq 0$ ). $\Delta S_{\infty \max }$ is the dimensionless thermodynamic maximum of the entropy increment in a random process without controlling factors of non-random nature; $\delta S_{d \infty}$ and $\delta S_{n \infty}$ are the asymptotic stationary increments of entropy on the day and night sides of the planet, taking into account its rotation. 
Thus, the second law of thermodynamics continues to govern at least for the considered class of non-isolated systems and random processes in them. This situation is shown in the graphs $T(t)$ and $\Delta S(t)$ in Figure 3, Figure 4. The asymptotic values of temperature and entropy are shown at $t \rightarrow \infty$, in a state of new thermodynamic equilibrium:

$$
\begin{aligned}
& T(t) \rightarrow T_{\infty}=1, \\
& \Delta S(T) \rightarrow \Delta S_{\infty}(1)= \frac{1}{2}\left(\ln 3-\ln \left(T_{0}^{2}+T_{0}+1\right)+2\left(\frac{1}{T_{0}}-1\right)\right. \\
&\left.-\frac{2}{\sqrt{3}}\left(\frac{\pi}{3}-\operatorname{arctg} \frac{1+2 T_{0}}{\sqrt{3}}\right)\right)>0 .
\end{aligned}
$$

\section{Accounting for Body Rotation Being a Regular Factor of Non-Random Nature}

Before you begin to format your It is possible to lower the entropy of the body to values less than its thermodynamic maximum by introducing a regular factor into the system, for example, periodically interrupting the ingoing heat flow $P_{s}(t)=P_{s}(t+\tau)$ in the considered example, taking into account the rotation of the planet around its axis (Figure 2, $\tau$ is the planet's day length (day + night)).

\section{The Night Side of the Planet}

On the day side, Equation (11), Equation (12) and their solutions (16), (17) remain valid. There is no ingoing heat flow on the night side, therefore,

$$
P_{s}=0, \frac{\mathrm{d} S_{\text {inp }}}{\mathrm{d} t}=0, \frac{\mathrm{d} S_{\text {int }}}{\mathrm{d} t}=0, \frac{\delta A}{\mathrm{~d} t}=0,
$$

whence it follows from (2) (dimensional record):

$$
\frac{\mathrm{d} S}{\mathrm{~d} t}=\frac{\mathrm{d} S_{\text {ext }}}{\mathrm{d} t}=-\frac{\mathrm{d} S_{\text {out }}}{\mathrm{d} t}=-\frac{P(T)}{T}=-\Pi \sigma T^{3}=\frac{1}{T} C_{v} M \frac{\mathrm{d} T}{\mathrm{~d} t} .
$$

The dimensionless equations follow from (20):

$$
\begin{gathered}
-\alpha \mathrm{d} t=\frac{\mathrm{d} T}{T^{4}}, \\
\mathrm{~d} S=\frac{\mathrm{d} T}{T}
\end{gathered}
$$

And their solutions:

$$
\begin{aligned}
& T(t)=\left(3 \alpha\left(t-t_{*}\right)+T_{*}^{-3}\right)^{-\frac{1}{3}}, \\
& \Delta S=S(T)-S_{*}\left(T_{*}\right)=\ln \frac{T}{T_{*}},
\end{aligned}
$$

where $T_{*}$ and $S_{*}$ are the temperature and entropy at the beginning of the night at $t=t_{*}$. 


\section{The Decrease in Entropy When a Periodic Factor of Non-Random Nature Is in Effect}

Consider the temperatures $T_{d}$ and $T_{n}$ and the entropies $S_{d}$ and $S_{n}$ at the ends of the day and night sections (Figure 3, Figure 4, lower curves, the initial value $T_{0}$ is given):

Day: $2 n \leq t \leq 2 n+1, n=0,1,2, \cdots$

$$
\begin{aligned}
& T_{d 2 n+1}=\left(1+\left(T_{n 2 n}^{-3}-1\right) \mathrm{e}^{-3 \alpha}\right)^{-\frac{1}{3}}, \\
\Delta S_{d 2 n+1}= & S_{d 2 n+1}-S_{n 2 n} \\
= & \frac{1}{2}\left(\ln \frac{1+T_{d 2 n+1}+T_{d 2 n+1}^{2}}{1+T_{n 2 n}+T_{n 2 n}^{2}}+2\left(\frac{1}{T_{n 2 n}}-\frac{1}{T_{d 2 n+1}}\right) .\right. \\
& \left.-\frac{2}{\sqrt{3}}\left(\operatorname{arctg} \frac{2 T_{d 2 n+1}+1}{\sqrt{3}}-\operatorname{arctg} \frac{2 T_{n 2 n}+1}{\sqrt{3}}\right)\right) .
\end{aligned}
$$

Night: $2 n+1 \leq t \leq 2 n+2$

$$
\begin{gathered}
T_{n 2 n+2}=\left(3 \alpha+T_{d 2 n+1}^{-3}\right)^{-\frac{1}{3}}, \\
\Delta S_{n 2 n+2}=S_{n 2 n+2}-S_{d 2 n+1}=\ln \frac{T_{n 2 n+2}}{T_{d 2 n+1}} .
\end{gathered}
$$

In formulas (26)-(28), the first $n$ means "night", and the second $n \in 0,1,2, \cdots$ means integers.

$$
\begin{gathered}
\delta S_{d 2 n+1}=S_{d 2 n+1}-S_{0}=S_{n 2 n}+\Delta S_{d 2 n+1}-S_{0} \\
\delta S_{n 2 n+2}=S_{n 2 n+2}-S_{0}=S_{d 2 n+1}+\Delta S_{n 2 n+2}-S_{0} .
\end{gathered}
$$

Their dependence on time is shown in Figure 4.

At $n \rightarrow \infty$, a rotating planet changes to its new stationary state at constant day and night asymptotic temperatures $T_{d \infty}, T_{n \infty}$ and entropies $\delta S_{d \infty}, \delta S_{n \infty}$ :

$$
\begin{gathered}
T_{d \infty}=\left(1+\frac{3 \alpha \mathrm{e}^{-3 \alpha}}{1-\mathrm{e}^{-3 \alpha}}\right)^{-\frac{1}{3}}, \\
T_{n \infty}=\left(1+\frac{3 \alpha}{1-\mathrm{e}^{-3 \alpha}}\right)^{-\frac{1}{3}}, \\
\Delta S_{d \infty}=\frac{1}{2}\left(\ln \frac{1+T_{d \infty}+T_{d \infty}^{2}}{1+T_{n \infty}+T_{n \infty}^{2}}+2\left(\frac{1}{T_{n \infty}}-\frac{1}{T_{d \infty}}\right)\right. \\
\left.-\frac{2}{\sqrt{3}}\left(\operatorname{arctg} \frac{2 T_{d \infty}+1}{\sqrt{3}}-\operatorname{arctg} \frac{2 T_{n \infty}+1}{\sqrt{3}}\right)\right) \\
\Delta S_{n \infty}=\ln \frac{T_{n \infty}}{T_{d \infty}}<0 .
\end{gathered}
$$

Their graphs are presented in Figure 3, Figure 4. Two important results follow from these data: 
- The increments of temperature and entropy on the day side are always greater than the decreases in temperature and entropy on the night side; the increment of entropy for a period, that is, for one revolution, always grows;

- At $t \rightarrow \infty(n \rightarrow \infty)$, the stationary asymptotic temperature and entropy increments, $T_{d \infty}, T_{n \infty}$ and $\delta S_{d \infty}, \delta S_{n \infty}$ are less than their maximum equilibrium values in a purely random process.

In principle, if to extend the night state in time (pause when the body heats up) and reduce the daytime state in time, we can achieve a decrease in temperature, and therefore, entropy, according to the third law of thermodynamics (Nernst theorem [5]), down to zero. But we will not do this in this work, this is a separate task that requires complicating the model by taking into account the dependence of the heat capacity of the body on temperature and fulfilling the condition: at $t \rightarrow 0, \lim c_{v}(T)=0$. The results will not be affected by such a complication obtained in this model.

Using formulas (29), (30), we can calculate the value of the day and night asymptotic temperatures for three planets: Mercury, Mars, and Pluto, calculating the parameter $\alpha$ (13) for them from the known experimental values: the planet's revolution period $\tau$, the total radiation temperature due to solar radiation $T_{s}$ heat capacity $\mathcal{C}_{\mathrm{n}}$ density of matter on the surface of the planet $\rho$, and the depth of daily heating $h[7]$ :

$$
\alpha=\frac{\sigma \tau T_{s}^{3}}{2 c_{v} \rho h}
$$

The following values were selected as average: heat capacity 1000 Joule/kg K, soil density $5000 \mathrm{~kg} / \mathrm{m}^{3}$, depth of daily heating of the planet's surface $1 \mathrm{~m}$.

The results are presented in Table 1. It can be seen from it that the theoretical dimensional values of $T_{d \infty}, T_{n \infty}$ are in good agreement with their experimentally obtained values [7].

The asymptotic temperature $(t \rightarrow \infty)$ of Mercury on the day side $T_{d \infty}$ is equal to the total radiation temperature due to solar radiation $T_{s}$. This is due to the fact that the day of Mercury is long enough; it manages to come into a state of thermal equilibrium with radiation. And during the night, it manages to cool noticeably: the temperature difference on the day and night sides is $480 \mathrm{~K}$.

The days of Mars are relatively short. Therefore, the temperature difference is not so significant. The shorter the revolution period $\tau$, the smaller the difference in the dimensional temperatures $T_{d \infty}$ and $T_{n \infty}$. In this regard, the difference in day and night temperatures in Mars is small and is equal to $10 \mathrm{~K}$.

The length of the day in Pluto is several times longer than that of Mars, but the total radiation temperature due to solar radiation is much less due to the great remoteness of Pluto from the Sun. Because of this, its temperature practically does not change during its day.

\section{Discussion of Results}

In the world around, everything is ordered and expedient. Why so? Indeed, there 
are always random collisions in it of the particles forming it, knocking out individual atoms from the ordered structures. If we didn't take any action that would return randomly detached atoms to their place, then the world would, according to the second law, surely collapse, and turn into chaos or cosmic dust in a finite time.

However, this does not happen. This means that there are guarding and restoring forces in nature, always returning "fallen atoms" to their place, i.e. ensuring the stability of the existence of each object: an elementary particle, the nucleus of an atom, cell, planet, star, ... the universe as a whole.

What are these forces doing? What does the "existence" of an object mean? When it's possible? Can forces of random, thermodynamic origin do this? Most likely, they cannot, and for a very simple reason. Because these forces (thermal conductivity, viscosity, diffusion, etc.) are rough, many micro particles always fall into their coverage area. But "building" and "restoring" forces need the ability to act locally; they influence on each particle separately in order to either build an ordered structured object in conditions of random collisions, or to recreate an object that deforms under random collisions. Otherwise, an attempt to rearrange, say, one micro particle, would entail the collective movement of many other micro particles that fell into the zone of action of thermodynamic forces.

\section{Conclusions}

The main result that can follow from this simple thermodynamic model of non-isolated systems: the cause of a decrease in entropy as a measure of disorder in such systems, which makes it possible for the phenomenon of "self-organization" to occur (the appearance of such structures as, say, Benard cells, the Belousov-Zhabotinsky reaction [8] [9] [10], etc.) is not the random process itself of entropy removal by the outgoing heat flow in non-isolated systems. As shown in this paper, it actually leads to the increase in entropy and to achieve a thermodynamic maximum in temperature and entropy in equilibrium state, as it takes in isolated systems.

An "entropy pump", which was regarded in some studies on synergetics as acting due to purely random processes of exporting entropy from a non-isolated system, does not work if we take into account the increase in entropy of the ingoing heat flow when it is thermalized at the input during the scattering of photons on the crystal lattice of the body (planet) surface.

The reason for the decrease in entropy can be regular factors of non-random nature (for example, periodic interruption of the ingoing heat flow), which can ensure that the temperature and entropy achieved in the new stationary state the values below the thermodynamic maximum.

\section{Conflicts of Interest}

The authors declare no conflicts of interest regarding the publication of this paper. 


\section{References}

[1] Prigogine (1955) Introduction to Thermodynamics of Irreversible Processes. Charles C Tomas, Springfield, IL.

[2] Ebeling, V., Engel, A. and Feistel, R. (2001) Physics of the Evolution Processes. M., URSS, $35+68$.

[3] Prigogine, I. and Stengers, I. (1986) Order out of Chaos. A New Dialogue with Nature. M.: Progress, 432 p.

[4] Osipov, A.I. and Uvarov, A.V. (2004) Soros Educational Journal, 8, 70.

[5] Cooney, F.M. (1981) Statistical Physics and Thermodynamics. M., Nauka.

[6] Landau, L.D. and Lifshits, E.M. (2002) Statistical Physics. Part I. V.5, M .; Fizmatlit, $616 \mathrm{p}$.

[7] Vorontsov-Veliaminov, B.A. and Straut, E.N. (2004) Drofa. Astronomy.

[8] Rudenko, A.P. (1969) The Theory of Self-Development of Non-Isolated Catalytic Systems. M.: Publishing House of Moscow State University, 276 p.

[9] Gershuni, G.Z. and Zhukhovitsky, E.M. (1972) Convective Instability of an Incompressible Fluid. M.: Nauka, 5.

[10] Prigogine, I. and Defay, R. (1966) Chemical Thermodynamics. Nauka. Siberian Branch, Novosibirsk. 